\title{
Correlation between cycle threshold and viral load through comparison of RT-PCR qualitative versus quantitative assay for SARS-CoV-2
}

\author{
Angela Saraiello, Federica Ferrentino, Nunzia Cuomo, Maria Grimaldi, Erasmo Falco, \\ Marcello Raffone, Antimo Di Spirito, Nazareno Melillo, Gennaro Montanino, Valentino Guarino, \\ Francesco Nappo, Martina Esposito, Ilaria Cavallaro, Giovanni D’Auria, Luigi Atripaldi, Claudia Tiberio \\ Unità Operativa Complessa Microbiologia e Virologia, Ospedale Cotugno Azienda Ospedaliera dei Colli, Napoli, Italy
}

\section{Summary}

Background and aims. Real-time reverse transcription polymerase chain reaction (RT-PCR) is the gold-standard assay to

\footnotetext{
Correspondence: Claudia Tiberio, Unità Operativa Complessa Microbiologia e Virologia, Ospedale Cotugno Azienda Ospedaliera dei Colli, Napoli, Italy.

Tel.: +39.3355779708.

E-mail: claudia.tiberio@ospedalideicolli.it
}

Key words: SARS-CoV-2; Qualitative analysis; Quantitative analysis; Viral RNA load; Respiratory sample.

Acknowledgments: The authors would thank all teams at Cotugno Hospital involved in the care of patients with and without COVID-19. Further thanks to the Istituto Zooprofilattico Sperimentale del Mezzoggiorno (IZSM) for providing the samples on which to conduct the study, and product support.

Contributions: AS and CT wrote the manuscript; FF, MG and MR designed the study; NM, VG, FN, ME, GM, ADS, IC and GD performed the sample analysis; AS, EF and NC performed the statistical analysis; LA, CT and AS critically revised the manuscript. All authors have accepted responsibility for the entire content of the submitted manuscript and approved submission.

Conflict of interest: The authors declare no potential conflict of interest. Funding: None.

Ethics approval and consent to publication: The studies involving human participants were reviewed and approved by the Ethic Committee of the University Federico II; Dipartimento Di Scienze Biomediche Avanzate [President: Prof. Claudio Buccelli]". The patients/participants provided written informed consent to participate in this study.

Availability of data and materials: All data and materials are available by the corresponding authors.

Received for publication: 24 July 2021

Accepted for publication: 25 August 2021.

${ }^{\circ}$ Copyright: the Author(s), 2021

Licensee PAGEPress, Italy

Microbiologia Medica 2021; 36:9999

doi:10.4081/mm.2021.9999

This article is distributed under the terms of the Creative Commons Attribution Noncommercial License (by-nc 4.0) which permits any noncommercial use, distribution, and reproduction in any medium, provided the original author(s) and source are credited. detect SARS-CoV-2, but it has limitations compared to viral load analysis. Quantitative detection improves surveillance, diagnosis, and prevention. We performed a comparative study of qualitative and quantitative tests for the diagnosis of COVID-19 on respiratory samples from patients screened for SARS-CoV-2 infection, and explored the correlation between viral load compared to the threshold cycle $(\mathrm{Ct})$ value obtained in RT-PCR.

Materials and methods. Sixty respiratory samples from patients affected by SARS-CoV-2 were subjected to both the qualitative (Allplex TM 2019-nCoV Seegene) and the quantitative (Clonit ${ }^{\circledR}$ Quanty COVID-19) assays, and the relationship between viral load and $C t$ value was assessed by Spearman correlation analysis $(\rho)$. In addition, the viral load of samples collected from a patient with symptomatic cancer was monitored.

Results. The results show 100\% agreement between the results obtained with quantitative assay and the reference standards, whereas $99.2 \%$ agreement was found for the qualitative test. A strong negative Spearman's correlation between the $C t$ values of the $N$ genes and $R d R P$ gene was observed from qualitative assay values and viral loads.

Conclusions. Quantitative assay has a higher sensitivity than qualitative assay, and viral load testing allows the clinicians to better orient themself in the choice of therapeutic treatment to be adopted. The constantly higher viral load of clinical cases considered, irrespective of the different therapies used, confirms that viral load monitoring could represent a great advantage in clinical practice.

\section{Background}

The current sanitary emergency represented by COVID-19 led us to evaluate the diagnostic sensitivity of the new assays introduced for the detection of SARS CoV-2 in respiratory samples.

In this case, we compared the results of two molecular biology tests: qualitative and quantitative RT-PCR Assays.

For this purpose, 60 positive respiratory samples from patients affected by SARS-CoV-2 were subjected to both a serological test and molecular investigation at the municipality of ArianoIrpinousing a total of 650 subjects positive only for serological screening of antibodies. Of these 60 samples, 44 were reconfirmed positive and 16 negatives by the qualitative (Allplex ${ }^{\text {TM }}$ 2019-nCoV Seegene) assay at the molecular virology laboratory of the Cotugno Hospital. Since none of the positive cases analyzed showed any relevant clinical symptoms, it was decided to also carry out an evaluation of the viral load by testing the samples using the quantitative Clonit ${ }^{\circledR}$ Quanty Covid-19 kit. 
The comparison between the data obtained through the two assays made it possible to test their respective sensitivity.

The comparison between the two methods of testing also made it possible to evaluate the correlation between Threshold Cycle $(\mathrm{Ct})$ value and viral load and therefore also the clinical relevance of the qualitative method compared to quantitative.

In this respect, this study demonstrates a negative Spearman's correlation between the viral loads quantified by the RT-PCR quantitative assay and the $C t$ values obtained with qualitative assay. This correlation is particularly high for the $\mathrm{N}$ gene of the qualitative test $(\mathrm{N}$ gene, $\rho=-0.93$; RdRP gene, $\rho=-0.90$ ).

Furthermore, to evaluate the applicability of the method in the clinical monitoring of immunocompromised patients, we also performed a small prospective study to monitor, using the quantitative test, the viral load of samples collected from a symptomatic cancer patient admitted to Cotugno Hospital, in order to monitor the change in viral load following the different therapies used, thereby confirming a severe impairment of the immune system.

This made it clear that viral load monitoring, accompanied by larger comparative studies, could represent a great advantage in clinical practice.

\section{Introduction}

To date, the Coronavirus Disease 2019 (COVID-19) epidemic has become a health emergency of global concern, and Severe Acute Respiratory Syndrome Coronavirus 2 SARS-CoV-2 is the pathogen identified $[1,2]$. More than 82 million people are infected, with more than 1.8 million deaths in 235 countries, areas, or territories. In Italy, up to December 20201,825775 positive cases and 64,036 deaths were recorded, making Italy the seventh country in the world and the fourth in Europe for total number of cases and overall, the fifth country in the world and the first in Europe by number of deaths [3].

At present, Reverse Transcription-Polymerase Chain Reaction (RT- PCR) is the gold standard for the Qualitative diagnosis of COVID-19 [4], but it has limitations with respect to viral load analysis. This does not allow for an evaluation of prognosis, disease progression and most importantly the efficacy of therapy.

The sensitivity of RT-PCR has been questioned in the case of both negative results found in some patients (who were strongly suspected of having the disease) and positive results in some confirmed cases after recovery $[5,6]$.

This technique involves use of a threshold Cycle $(C t)$ value that cannot be directly interpreted as viral load without a standard curve using reference materials and can be correlated to the initial target concentration through fluorescence intensity in the sample; this is then used to determine only whether a sample is positive or negative [7].

For this reason, quantitative detection is important because through viral load it is possible to improve surveillance, diagnosis and prevention by providing an ad-hoc therapy for the patient COVID-19 [8].

To this end, we both performed a comparative study of qualitative and quantitative RT-PCR assay for the diagnosis of COVID19 on respiratory samples from patients screened for SARS-CoV2 infection, and explored the changes in the viral load of asymptomatic patients through the $C t$ value obtained by qualitative assay.

We also evaluated the applicability of the quantitative assay in the clinical monitoring of immunocompromised patients, through a clinical case of a symptomatic cancer patient.

\section{Materials and Methods}

This study was done on respiratory samples of patients with confirmed COVID19 admitted to Cotugno Hospital emergency ward during a period in May 2020.The entire evaluation work took place in the period between July and October 2020.

All samples tested positive for SARS-CoV-2 RNA by the qualitative assay were eligible for the study. Among these positive samples, we then selected some with different cycle threshold values $(C t)$, therefore representative of different levels of positivity.

All the samples were processed for qualitative (Allplex ${ }^{\mathrm{TM}}$ 2019nCoV Seegene) assay with a Seegene Nimbus system (Arrow Diagnostics), which performs PCR setup and RNA extraction. Subsequently the Real Time PCR amplification reaction was set up through the CFX96 Touch system. Finally, the results were automatically interpreted and analyzed by the Seegene Viewer 2019- nCoV software. Table 1 shows that a positive result predicts the presence of all three viral target genes (RdRP, E and $\mathrm{N}$ genes). The detection of at least one of the two specific viral targets indicates the presence of SARS-CoV-2 RNA in the patient sample with certainty, while the $\mathrm{E}$ gene alone indicates the presumed presence of the viral genetic material in the patient sample. The table also indicates the fluorophores used corresponding to the different target gene.

The same patient samples, used for the qualitative assay, were then subjected to quantitative investigation using the quantitative (Clonit ${ }^{\circledR}$ Quanty Covid-19) assay. For this assay, the Sample's RNA extract obtained through MagNa Pure Compact (Roche Diagnostics) system extraction step was used.

Table 1. Interpretation of results through the kit Allplex ${ }^{\mathrm{TM}} 2019-\mathrm{nCoV}$ assay.

\begin{tabular}{|c|c|c|c|c|c|}
\hline Case & $\begin{array}{c}\text { IC } \\
(\mathrm{HEX})\end{array}$ & $\begin{array}{l}\text { E gene } \\
\text { (FAM) }\end{array}$ & $\begin{array}{c}\text { RDRPgene } \\
\text { (Cal Red 610) }\end{array}$ & $\begin{array}{c}\text { N gene } \\
\text { (Quasar 670) }\end{array}$ & $\begin{array}{l}\text { Interpretation by } \\
\text { Seegene Viewer }\end{array}$ \\
\hline 1 & $+/-$ & + & + & + & 2019-nCoVDetection \\
\hline 2 & $+/-$ & + & - & + & 2019-nCoVDetection \\
\hline 3 & $+/-$ & + & + & - & \\
\hline 4 & $+/-$ & - & + & + & \\
\hline 5 & $+/-$ & - & - & + & \\
\hline 6 & $+/-$ & - & + & - & \\
\hline 7 & $+/-$ & + & - & - & Presumptive positive \\
\hline 8 & + & - & - & - & Not detected \\
\hline 9 & - & - & - & - & Not valid \\
\hline
\end{tabular}


The presence of SARS-CoV-2 RNA in a patient can be confirmed if $\mathrm{N} 1, \mathrm{~N} 2$ and $\mathrm{N} 3$ genes are detected. If these genes are not detected the result is inconclusive. With regards to RNA quantitative detection of SARS-COV-2, we have a standard curve obtained following amplification ( $10^{1}$ to $10^{5}$ copies/ $\mu$ l of synthetic viral N1encoding RNA). So, we calculate the viral load interpolating the $C t$ values with the standard curve. Viral load (copies $/ \mathrm{ml}$ ) is the result of: $\mathrm{n} *(1000 / \mathrm{Ve}) *(\mathrm{Ev} / \mathrm{Ea})$. Where $\mathrm{n}$ is the number of viral copies, $\mathrm{Ve}$ is the volume of the extracted sample $(400 \mu \mathrm{l})$, Ev is the volume of the eluate $(50 \mu \mathrm{l})$ and $\mathrm{Ea}$ is the volume of the extract $(5 \mu \mathrm{l})$. Table 2 shows the evaluation criteria for Quanty COVID19 assay.

We performed a Spearman's correlation on all samples where the SARS-CoV-2 N1 gene concentration was between $10^{1}$ and $10^{7}$ copies $/ \mathrm{mL}$, in order to assess the correlation between Quanty COVID-19 viral load levels and Allplex TM 2019-nCoV test values.

\section{Results}

Following the outbreak in the municipality of ArianoIrpino in May, an extensive serological screening was carried out which revealed that out of 650 positive subjects for antibody research, 60 were positive for Sars-CoV-2 with nose-pharyngeal swab.

From ArianoIrpino the positive samples were sent to Naples for a counter-analysis at the virology and microbiology laboratory of the Cotugno Hospital where, out of a total of 60 samples, 44 were confirmed positive.

Since all the positive cases analyzed did not show any relevant clinical symptoms, it was decided to also carry out an evaluation of the viral load by testing the samples using the Clonit kit.

Table 3 shows the results of either positive (44) and negative (16) with the Allplex 2019-nCoV assay for a total of 60 samples. Then, we compared these results with those of the Quanty COVID19 assay. As shown in Table 3, the Allplex 2019-nCoV positive samples $C t$ values of $\mathrm{E}, \mathrm{RdRP}$, and $\mathrm{N}$ genes are between 23.8 and 35.5 (24 samples), RdRP and $\mathrm{N}$ genes (15 samples) are between 34.1 and 39.1, while $\mathrm{N}$ gene ( 5 samples) are between 37.5 and 38.1 .

We evaluated viral load values through the Quanty COVID19 of positive samples and we show the results on the Table 4. In samples with viral load levels from $>2.0$ to $\leq 3.0$ or $>1.0$ to $\leq 2.0 \log 10$ copies per $\mathrm{ml}$, our results shown detections of $\mathrm{N}$ gene $(54.5 \%$ and $41.0 \%$, respectively), RdRP gene ( $18.0 \%$ and $16.0 \%$, respectively),

Table 2. Results evaluation criteria for Quanty COVID19 assay. A sample is positive for SARS- COV-2 if fluorescence is detected in all three targets $(\mathrm{N} 1, \mathrm{~N} 2$ and $\mathrm{N} 3)$. If one or more targets are negative, however, the data is inconclusive.

\begin{tabular}{lcccc} 
N1 (FAM) & N2 (VIC) & N3 (VIC) & RP (Cy5) & Results \\
+ & + & + & $<35$ & Sars-CoV-2 Positive \\
- & - & - & $<35$ & Sars-CoV-2Negative \\
\hline- & - & - & $>35$ & Invalid result \\
$1-2$ positive targets & & & $<35$ & Inconclusive result \\
\hline
\end{tabular}

N: Nucleocapsid; RP: Ribonucleasi P (internal endogenous control); VIC: 2 -chloro-7 phenyl-1,4-dichloro-6-carboxy-fluorescein; Cy5: Cyanine 5.

Table 3. Results for 60 respiratory samples tested by SARS-CoV-2 detection assays.

\begin{tabular}{lcc} 
Seegene, samples (CT range) & $\begin{array}{c}\text { Cloniti( Quanty Covid-19, } \\
\text { samples (CT range) }\end{array}$ \\
Positive results & $44(17.9-39.4)$ & $44(18.7-39.8)$ \\
Targets Genes & $24(23.8-35.5)$ \\
N, E, RdRP & $15(34.1-39.1)$ & \\
N and RdRP & $5(37.5-38.1)$ & \\
N & & $44(18.7-39.8)$ \\
N1, N2, N3 & $16(0.0-0.0)$ & $0(0)$ \\
\hline Negative results &
\end{tabular}

N: nucleocapsid; E: Envelope; RDRP: RNA dependent RNA polimerase.

Table 4. Results obtained from the Allplex 2019-nCoV method based on the viral load ranges obtained by quantitative assay for positive respiratory samples.

\begin{tabular}{|c|c|c|c|}
\hline Viral load levels & Number of dete & plex TM 201 & ene targets $(\%)^{*}$ \\
\hline$(\log 10$ copies/ml) & N gene & RdRP gene & E gene \\
\hline$\leq 1.0$ & $0(0)$ & $0(0)$ & $0(0)$ \\
\hline$>1.0-\leq 2.0$ & $18(41)$ & $4(17)$ & $16(88.8)$ \\
\hline$>2.0-\leq 3.0$ & $24(54.5)$ & $17(70.6)$ & $1(5.6)$ \\
\hline$>3.0-\leq 4.0$ & $1(2.25)$ & $3(12)$ & $0(0.0)$ \\
\hline$>4.0-\leq 5.0$ & $0(0)$ & $1(0.4)$ & $1(5.6)$ \\
\hline$>5.0-\leq 6.0$ & $1(2.25)$ & $0(0)$ & $0(0.0)$ \\
\hline Total & 44 & 25 & 18 \\
\hline
\end{tabular}

*The Allplex 2019-nCoV targets the E (envelope), RdRP (RNA-dependent RNA polymerase), and N (nucleocapsid) genes of SARS-CoV-2. 
and $\mathrm{E}$ gene $(5.5 \%$ and $88.8 \%$, respectively). The relationship between viral load and $\mathrm{Ct}$ value was evaluated through a Spearman's correlation $(\rho)$ analysis. A strong negative association between the Ct Values of Ngenes $(\rho=-0.93$; $<0.001)$ and RdRP $(\rho=-0.90 ; \quad P<0.001)$ was observed from Allplex 2019-nCoV assay's values and viral loads compared to E gene (Figures 1-4).

\section{Discussion}

The laboratory-based diagnosis for COVID-19 is changing rapidly [9]. It is therefore necessary to determine the accuracy of every new assays for SARS-CoV-2. Since December 2019 the challenge has been to use tests that can ensure reliable and rapid detection of the virus also because early diagnosis of SARS-CoV2 is important in the prevention and control during this pandemic. It is useful, therefore, to have a molecular target which is known to be more valid than others, in order to redesign these assays (for example, in order to move from a single target to multiple, specific targets), and to make more informed choices relating to which tests to use in clinical laboratories.

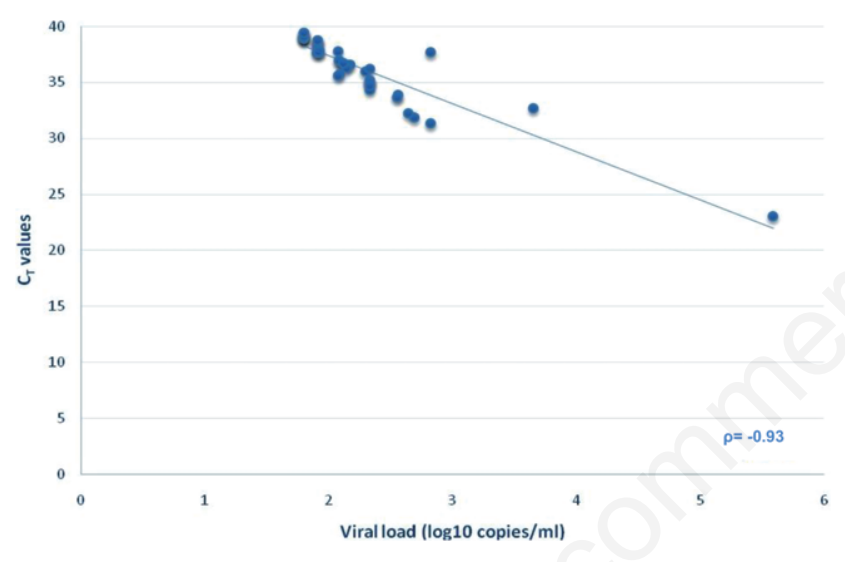

Figure 1. The figure shows the viral load levels from Quanty COVID 19 assay and the Ct values of Allplex 2019-nCoV assay for SARS-CoV-2 $\mathrm{N}$ gene.

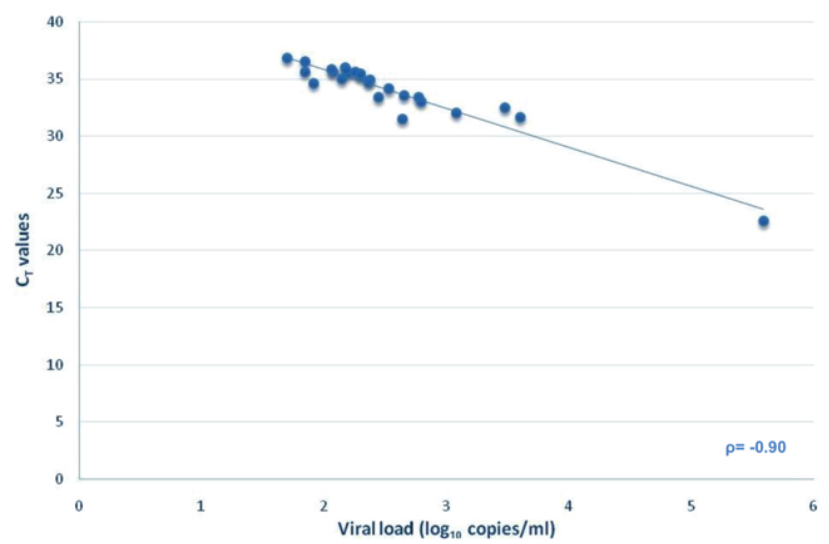

Figure 2. The figure shows the viral load levels from Quanty COVID 19 assay and the Ct values of Allplex 2019-nCoV assay for SARS-CoV-2 RDRP gene.
The RT-PCR, among all available tests, is considered the gold standard thanks to its advantages as a specific and simple quality test. However, the RT-PCR method has an important limitation: it does not provide precise indications on viral load, necessary to evaluate the progression of the disease and prognosis. Testing the viral load through a quantitative dosage of the pathogen's genome allows the clinician to better orient themself in the choice of therapeutic treatment to be adopted, providing them with the possibility to follow the evolution of anti-viral therapy and the follow-up of the patient.

This is fundamental data at the diagnostic level as the outcome of SARS-CoV-2 infection is decided between 10-15 days of infection and is directly dependent on viral load. For example, a positive patient just above the cut-off will have a completely different clinical course from a patient with a million viral copies per microliter, just as a positive result of RT-PCR doesn't necessarily mean that the patient is infectious or that he has a significant disease, in fact, the amount of vital virus may be too low for transmission. The progression of SARS-CoV-2 disease is characterized by a non-linear dynamic of virulence; therefore, a high viral load does not always reflect a serious clinical condition.

However, knowledge of the viral load can at least give an indi-

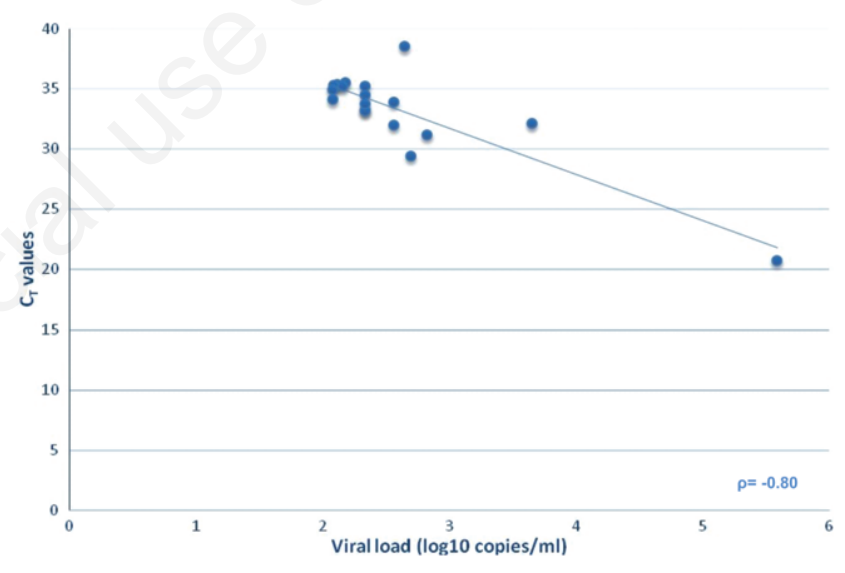

Figure 3. The figure shows the viral load levels from Quanty COVID19 assay and the Ct values of Allplex 2019-nCoV assay for SARS-CoV-2 E gene.

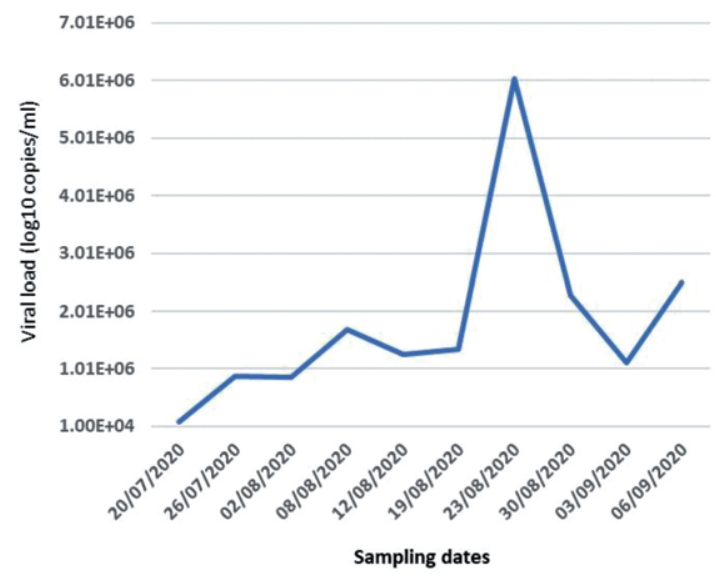

Figure 4. Results of the quantitative test carried out on the samples of the clinical case monitored. 
cation of the transmissibility of the pathogen [10]. The diagnosis of SARS-CoV-2 implements in parallel with the need to have rigorous systems that guarantee rapid and accurate detection of the virus, and $\mathrm{Ct}$ value cannot be directly interpreted as viral load without a standard curve using reference material [11].

The objective of our work was both to test the sensibility of the qualitative method compared to the quantitative one and also to evaluate, through this comparison, the relative usefulness of the two methods to clinicians through the correlation between Threshold Cycle $(\mathrm{Ct})$ value and viral load.

Today, the interpretative criteria on qualitative assay [12] consider that a sample is positive to SARS-CoV-2 if one of the 3 specific target genes is positive. In all 44 samples positive, the presence of the $\mathrm{N}$ gene was found. Namely, the quantitative assay uses the $\mathrm{N}$ gene as the only molecular target, supporting the US CDC to consider gene $\mathrm{N}$ as the only target gene for the diagnosis of SARS-CoV-2 [13].

We used qualitative assay to evaluate the SARS-CoV-2 RNA in 60 respiratory samples considered for this research, later used the quantitative assay to quantify SARS-CoV-2 RNA in the 44 positive samples of the 60 total respiratory samples taken into consideration, and the same extract was used for the qualitative and quantitative analysis.

The results show 100\% agreement between the results obtained with quantitative assay and the reference standards, while for the qualitative assay we found a $99.2 \%$ agreement. Specifically, we observed that some false negative samples for the qualitative test, when subsequently subjected to quantification, were positive on quantitative assays with N2 gene from 37.2 to 38 , and N3 gene from 37.8 to 39 [14]. However, it has been shown that there is a slight difference in the correlation between the various assays, and in particular that the quantitative test proved to be more sensitive.

To evaluate the relationship between $\mathrm{Ct}$ value e viral load Spearman's correlation was used, which revealed both that, in line with studies, the virulence of a sample is inversely proportional to the $C t$ value of the RT-PCR performed with the qualitative test [15$18]$, and also that a negative Spearman's correlation is particularly high for the $\mathrm{N}$ gene of the qualitative test.

All samples from the ArianoIrpino outbreak showed considerable viral load, and this confirms the possibility of transmission of the disease by asymptomatic or minimally symptomatic patients.

It was also essential to evaluate the applicability of the quantitative assay in the clinical monitoring of immunocompromised patients, by monitoring the infection of the clinical case. The results obtained demonstrate the presence of a viral load, which is certainly variable but consistently higher than the initial values, which testifies to a severely compromised immune system. Unfortunately, the patient died, despite the therapy that lasted the entire course of the pathology.

\section{Conclusions}

In conclusion, to monitor infection of SARS-CoV-2, the quantitative assay can be extremely useful. However, it may be useful to investigate further to define whether these types of assays might be used in the future. Of course, as the testing for COVID-19 increases, these assays can contribute to boosting the laboratory tests of SARS-CoV-2 infection.

Considering the observations of the results obtained, to date, at the request of clinicians, the quantitative test has been introduced in the routine laboratory tests to monitor the course of the disease.

\section{References}

1. Harcourt J, Tamin A, Xiaoyan L, et al. Severe Acute Respiratory Syndrome Coronavirus 2 from Patient with Coronavirus Disease, United States. EID J 2020:26.

2. Wu F, Zhao S, Yu B, et al. A new coronavirus associated with human respiratory disease in China. Nature 2020;579:265-9.

3. Curiale S. Gli anticorpi neutralizzanti contro il SARS-CoV-2 nei pazienti convalescenti sono ancora attivi a undici mesi dall'infezione. Available from: https://www.inmi.it/gli-anticorpi-neutralizzanti-contro-il-sars-cov-2-nei-pazienti-convalescenti-sono-ancora-attivi-a-undici-mesi-dallinfezione.html

4. World Health Organization. Laboratory testing for 2019 novel coronavirus (2019-nCoV) in suspected human cases. Available from: https:/www.who.int/publications-detail/laboratory-testing-for-2019- novelcoronavirus-in-suspected-human-cases20200117

5. Winichakoon $P$, Chaiwarith R, Liwsrisakun C, et al. Negative Nasopharyngeal and Oropharyngeal Swab Does Not Rule Out COVID-19. J Clin Microbiol 2020;58:e0297-20.

6. Wu J, Liu J, Zhao X, et al. Clinical Characteristics of Imported Cases of COVID-19 in Jiangsu Province: A Multicenter Descriptive Study. Clin Infect Dis 2020;71:706-12.

7. Chu DKW. Pan Y, Cheng SMS, Hui KPY, et al. Molecular diagnosis of a novel coronavirus (2019-nCoV) causing an outbreak of pneumonia. Clin. Chem 2020;66:549-55.

8. Yu F, Yan L, Wang N, et al. Quantitative detection and viral load analysis of SARS-CoV-2 in infected patients. Clin Infect Dis 2020;71:793-8.

9. Patel R, Babady E, Theel ES, et al. Report from the American Society for Microbiology COVID-19 international summit, 23 March 2020 Value of diagnostic testing for SARS-CoV2/COVID-19. mBio 2020;11:e00722-20.

10. Liu Y, Yan LM, Wan L, et al. Viral dynamics in mild and severe cases of COVID-19. Lancet Infect Dis 2020;20:656-7.

11. Han MS, Byun JH, Cho Y, Rim JH. RT-PCR for SARS-CoV2: quantitative versus qualitative. Lancet Infect Dis 2021;21:165.

12. Posteraro B, Marchetti S, Romano L, et al. Clinical microbiology laboratory adaptation to COVID-19emergency: experience at a large teaching hospital in Rome, Italy. Clin Microbiol Infect 2020;26:1109-11.

13. Holshue ML, DeBolt C, Lindquist S, et al. First case of 2019 novel coronavirus in the United States. N Engl J Med 2020;382:929-36.

14. Zhen W, Manji R, Smith E, Berry GJ. Comparison of four molecular in vitro diagnostic assays for the detection of SARSCoV-2 in nasopharyngeal specimens. J Clin Microbiol 2020;58:e00743-20.

15. Pan Y, Zhang D, Yang P, et al. Viral load of SARS-CoV-2 in clinical samples. Lancet Infect Dis 2020;20:411-2.

16. To KK, Tsang OT, Leung WS, et al. Temporal profiles of viral load in posterior oropharyngeal saliva samples and serum antibody responses during infection by SARS-CoV-2: an observational cohort study. Lancet Infect Dis 2020;20:565-74.

17. Wang W, Xu Y, Gao R, et al. Detection of SARS-CoV-2 in different types of clinical specimens. JAMA 2020;323:1843-4.

18. Zou L, Ruan F, Huang M, et al. SARS-CoV-2 viral load in upper respiratory specimens of infected patients. N Engl J Med 2020;382:1177-9. 